\title{
Application of a hierarchical enzyme classification method reveals the role of gut microbiome in human metabolism
}

\author{
Akram Mohammed', Chittibabu Guda ${ }^{1,2,3,4^{*}}$ \\ From The International Conference on Intelligent Biology and Medicine (ICIBM) 2014 \\ San Antonio, TX, USA. 04-06 December 2014
}

\begin{abstract}
Background: Enzymes are known as the molecular machines that drive the metabolism of an organism; hence identification of the full enzyme complement of an organism is essential to build the metabolic blueprint of that species as well as to understand the interplay of multiple species in an ecosystem. Experimental characterization of the enzymatic reactions of all enzymes in a genome is a tedious and expensive task. The problem is more pronounced in the metagenomic samples where even the species are not adequately cultured or characterized. Enzymes encoded by the gut microbiota play an essential role in the host metabolism; thus, warranting the need to accurately identify and annotate the full enzyme complements of species in the genomic and metagenomic projects. To fulfill this need, we develop and apply a method called ECemble, an ensemble approach to identify enzymes and enzyme classes and study the human gut metabolic pathways.
\end{abstract}

Results: ECemble method uses an ensemble of machine-learning methods to accurately model and predict enzymes from protein sequences and also identifies the enzyme classes and subclasses at the finest resolution. A tenfold cross-validation result shows accuracy between 97 and $99 \%$ at different levels in the hierarchy of enzyme classification, which is superior to comparable methods. We applied ECemble to predict the entire complements of enzymes from ten sequenced proteomes including the human proteome. We also applied this method to predict enzymes encoded by the human gut microbiome from gut metagenomic samples, and to study the role played by the microbe-derived enzymes in the human metabolism. After mapping the known and predicted enzymes to canonical human pathways, we identified 48 pathways that have at least one bacteria-encoded enzyme, which demonstrates the complementary role of gut microbiome in human gut metabolism. These pathways are primarily involved in metabolizing dietary nutrients such as carbohydrates, amino acids, lipids, cofactors and vitamins.

Conclusions: The ECemble method is able to hierarchically assign high quality enzyme annotations to genomic and metagenomic data. This study demonstrated the real application of ECemble to understand the indispensable role played by microbe-encoded enzymes in the healthy functioning of human metabolic systems.

\section{Background}

Enzymes represent a significant fraction of an individual proteome [1] and catalyze a variety of specific reactions in the cellular systems [2,3]. Hence, identification of the functions of an entire complement of enzymes in an

\footnotetext{
* Correspondence: babu.guda@unmc.edu

'Department of Genetics, Cell Biology and Anatomy, University of Nebraska Medical Center, Omaha, NE, 68198, USA

Full list of author information is available at the end of the article
}

organism helps generate the metabolic blueprint of that species. This will not only improve our understanding of defined cellular processes of individual species but also help study the metabolic interdependence of multiple species in an ecosystem such as the human gut microbiome.

The Enzyme Commission (EC) [4] has classified all enzymes based on the enzymatic reactions they catalyze. Each enzyme has an EC number, which is a hierarchical 
number that distinguishes enzymes by the type of reactions they catalyze. The EC groups all enzymes into six broad classes that include (1) oxidoreductases - catalyze oxidoreduction reactions; (2) transferases - catalyze the transfer of a chemical group from a donor to an acceptor; (3) hydrolases - catalyze the hydrolysis of various bonds; (4) lyases - enzymes that cleave bonds by means other than by hydrolysis; (5) isomerases - catalyze geometrical or structural changes within one molecule; and (6) ligases - catalyze the joining of two molecules coupled with hydrolysis of a pyrophosphate bond in ATP or a similar triphosphate. The EC classification system assigns a unique four-field number to each enzymatic activity (such as EC 1.2.1.3 for aldehyde dehydrogenase (NAD+)) where, the first three numbers (a.k.a. levels) of an EC number represent progressively finer description of the enzymatic reaction, while the last level mostly represents substrate specificity of a reaction $[5,6]$.

Experimental characterization of the enzymatic reactions of all enzymes in a genome or a metagenome is a tedious and expensive task. With the exception of a few well-characterized genomes such as Escherichia coli (E. coli) and yeast, the fraction of experimentally annotated enzymes in many sequenced genomes is very small. The problem is more pronounced in the metagenomic samples where even the species are not adequately cultured or characterized. To address this problem, computational approaches, which can build accurate models from known data to predict the unknown data, have been employed. Such models have been widely used to predict protein functions and annotate newly sequenced genomes [7-10].

Several computational methods exist for predicting enzyme classes (refer to our review [11]). Many of them use electronically inferred annotations by transferring the annotations (EC number, enzyme name and the reactants) of a known enzyme to an unknown enzyme, if the features of unknown match with the known. Most of the methods differ by the type of features they use to match proteins, which broadly include amino acid composition [12], sequence or structure homology [13-15], domain composition [16,17] and sequence motifs [18]. A variety of machine learning (ML) and data mining algorithms, including nearest-neighbor methods [19], support vector machines (SVMs) [20,21], Bayesian [22,23] and ensemble approaches $[24,25]$ have been employed to build models for enzyme classification. The performance of these methods varies based on the classification algorithm, input datasets and features used for model building. Nevertheless, most of the existing methods fail to predict EC levels 3 and 4 due to the increasing difficulty in predicting the finer levels in the hierarchy, thus offer only limited value to enzyme annotations. Many enzyme prediction methods exhibit a lack of balance between specificity and sensitivity; EzyPred [17] and EFICAz2.5 [24] offer limited sensitivity for certain enzyme classes and/or EC levels and run extremely slow with high false positive rates. Hence, the existing methods are inadequate for annotation of high-throughput sequences generated from genomic and metagenomic projects, warranting the need for developing a new computational method.

The current study requires the identification of all enzymes encoded by the gut microbiome in human because the enzymes encoded by the gut microbiota play an extensive role in the human metabolism. Human gut microbiome is the largest and most complex of all microbial communities that harbor human body, with a gene set that is about 150 times larger than that of the human gene set [26]. Human gut microbiome alone is estimated to contain about 1000-1500 different species [26,27], but a majority of them are yet to be characterized. These bacterial communities extensively contribute to human gut metabolism by complementing enzymes that are not encoded by the human genome, but are essential for digestion of complex polysaccharides, absorption, metabolism of amino acids and vitamins, shaping of the immunological environment and a wide range of other metabolic functions [28-30]. Changes in the composition of human microbiota have been linked to health conditions such as inflammatory bowel disease (IBD), antibiotic-resistant infections, obesity, colon cancer, symptomatic atherosclerosis and diabetes [31-33]. Hence, the identification and functional characterization of gut microbial enzymes is a very important step towards understanding the microbedependent component of the human metabolism.

In this study, we developed a new hierarchical enzyme classification method based on machine learning that accurately predicts if a protein sequence is an enzyme or a non-enzyme, and if an enzyme, what is the specific enzymatic reaction (class and subclasses) it carries out. We apply this method to identify the full enzyme complements of 10 sequenced genomes of model organisms, and also those of the microbial species in the metagenomic samples obtained from human gut microbiomes. The methodology developed in this project and its application to identifying the full complements of microbial enzymes has made it feasible to study the role of microbe-complemented enzymes in the human gut metabolism. To our knowledge, this study represents a novel and robust approach to studying the pathway level hostpathogen interactions in the human gut metabolism.

\section{Results and discussion}

We describe the results and discussion in two separate sections that include the method development followed by its application to study the pathways in human gut metabolism. Figure 1 shows a schematic of the method and its application. An ensemble of five different 


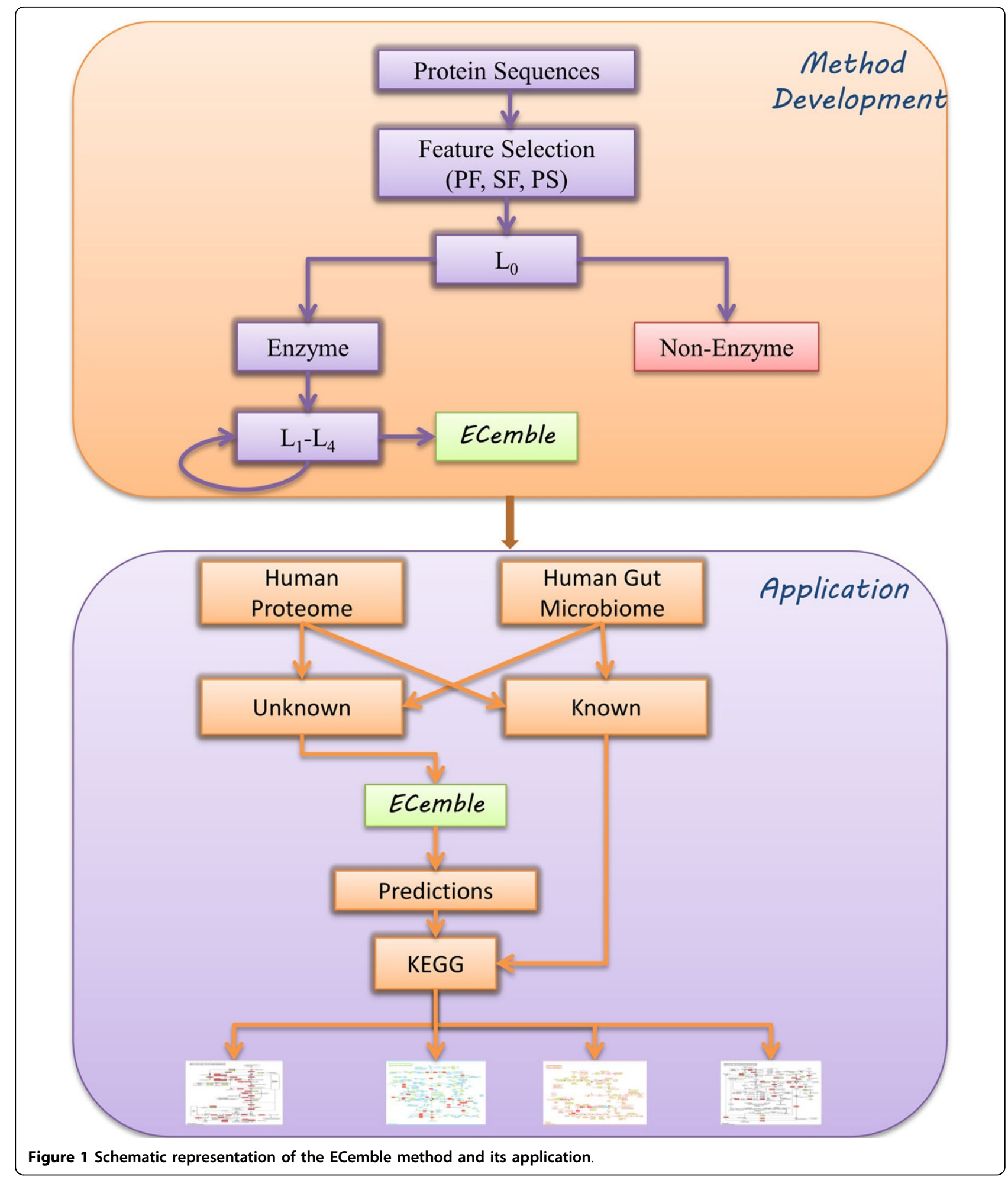

machine-learning (ML) classifiers was used to build prediction models based on protein domains that include sequence or structure-derived features. Hence, this method is named as ECemble (Enzyme Classification using ensemble approach). Bayesian network [22] model represents a set of random variables and their conditional dependencies, whereas Naïve Bayes [34] works under the strong assumption that there is independence 
among the features. K-nearest neighbor (KNN) [35] is 'instance based' learning algorithm. Despite its simplicity, it can offer very good performance. We tried polynomial and linear kernels in SVM [36] and polynomial kernel was found to be working better than the linear kernel. Random Forest classifiers (RFC) [37] work by generating large number of decision trees in a specific random way such that each one is de-correlated with the others. Each classifier model generates a probability distribution for all classes for each instance in the train and test set and the class with the highest probability is assigned as the predicted class.

This is a hierarchical prediction method that predicts enzymes and enzyme classes at 5 levels (henceforth referred to as $\mathrm{L}_{0}, \mathrm{~L}_{1}, \mathrm{~L}_{2}, \mathrm{~L}_{3}$ and $\mathrm{L}_{4}$ ), where the first model at $\mathrm{L}_{0}$ predicts if a protein is an enzyme or a non-enzyme, and the subsequent models from $\mathrm{L}_{1}$ to $\mathrm{L}_{4}$ predict specific class and subclass of an enzyme in the EC number hierarchy. The predictions are selected by a consensus approach, i.e., only when at least two of the three top-performing ML classifiers show consistent predictions. To demonstrate its usefulness, we applied this method to predict the full complement of enzymes from ten sequenced proteomes. In addition, we also tested about 2.5 million protein sequences that were obtained from metagenomic sequencing and assembly of the human gut microbiome [26]. Using the full complements of enzymes from human and human gut microbiomes, we further investigated the role of microbe-derived enzymes in the human gut metabolism.

\section{Development of ECemble method Feature selection and optimization}

We collected the known enzyme and non-enzyme protein sequences as positive and negative datasets, respectively. Sequences were clustered at $70 \%$ identity to generate 64,948 enzyme and 128,292 non-enzyme sequences, where each sequence has mapping to at least one of the following databases. These sequences were mapped against three domain databases that include Pfam [38], Superfamily [39] and Prosite [40] to extract the enzyme-specific and non-enzyme-specific features (as discussed in the Methods section). A protein domain is a conserved part of a protein's sequence or structure that can evolve, function, and exist independently of the rest of the protein chain. We used three domain databases that include Pfam, Superfamily and Prosite to represent the functional, structural and motif or active site regions of protein domains, respectively. These domains are used as features, where, in combination they offer a comprehensive feature set for ML methods to discriminate between enzymes and non-enzymes. We extracted 5,045 and 9,196 overlapping features for enzyme and nonenzyme datasets, respectively, from three domain databases (Table 1).
Table 1. Distribution of enzyme and non-enzyme features

\begin{tabular}{lcccc}
\hline Feature Set & Pfam & $\begin{array}{c}\text { Prosite } \\
\text { (PS) }\end{array}$ & $\begin{array}{c}\text { Superfamily } \\
\text { (SF) }\end{array}$ & $\begin{array}{c}\Sigma \text { (Pfam, } \\
\text { PS, SF) }\end{array}$ \\
\hline Database & 12273 & 1308 & 1962 & 15543 \\
Enzyme Only & 859 & 133 & 111 & 1103 \\
$\begin{array}{l}\text { Non-Enzyme Only } \\
\text { *Common }\end{array}$ & 7658 & 525 & 1013 & 9196 \\
$\begin{array}{l}\text { features } \\
\begin{array}{l}\text { Total unique } \\
\text { features }\end{array}\end{array}$ & 11031 & 1305 & 1905 & 14241 \\
\hline
\end{tabular}

*Common between enzyme and non-enzyme sequences

To facilitate the building of an effective ML-based classifier, it is essential to represent the domain features of each sequence in a binary format (feature vector), using ' 1 ' for the presence and ' 0 ' for the absence of a domain. The size of the feature vector matrix is $\mathrm{N} \times \Sigma(\mathrm{P}, \mathrm{Q}, \mathrm{R})$, where $\mathrm{N}$ is the total number of enzyme and non-enzyme sequences, and $\mathrm{P}, \mathrm{Q}$ and $\mathrm{R}$ represent the total number of mapped domains in Pfam, Superfamily and Prosite, respectively (Table 2 ). In theory, the dimensionality of this vector gets very large; however, since each protein sequence contains only a few domains, we used the sparse-formatting option by storing the occurrence of features with their locations in the feature space to significantly speed up the model building process.

\section{Hierarchical design of prediction models}

We applied five different machine-learning (ML) algorithms to best exploit features from the training dataset and optimize at each level. These include Naïve Bayes, k-Nearest Neighbor (KNN) classifier, Support Vector Machine (SVM), Decision Stump (DS) [41] and Random Forest (tree-based) classifiers (RFC). ML algorithms are employed to learn discriminative features of classes from the training data, build models, and test how related the unknown instances (testing data) are to these models. We used the WEKA (Waikato Environment for Knowledge Analysis) [42] framework to build prediction models in an iterative fashion at 5 different levels $\left(\mathrm{L}_{0}\right.$ to $\left.\mathrm{L}_{4}\right)$. As part of the enzyme identification step, the first model at $\mathrm{L}_{0}$ predicts if a protein sequence is an enzyme or not. Only the sequences predicted as enzymes at $\mathrm{L}_{0}$ are forwarded to build models for predicting enzyme classes

Table 2. Feature vectors and dimensionality for the dataset

\begin{tabular}{lcc}
\hline Dataset & $\begin{array}{c}\text { Number of feature } \\
\text { vectors }\end{array}$ & $\begin{array}{c}\text { Data } \\
\text { dimensionality }\end{array}$ \\
\hline Enzyme & 64948 & $5045 \times 64948$ \\
$\begin{array}{l}\text { Sequences } \\
\text { Mixed }\end{array}$ & 193240 & $14241 \times 193240$ \\
\hline
\end{tabular}

${ }^{\$}$ Both enzyme and non-enzyme sequences 
and subclasses at $\mathrm{L}_{1}-\mathrm{L}_{4}$, sequentially. At $\mathrm{L}_{0}$, there are only two classes (enzyme vs. non-enzyme) and similarly, at $\mathrm{L}_{1}$, there are only 6 enzyme classes; hence, one model is sufficient to predict classes at these two levels. However, the six enzyme classes at $L_{1}$ are further divided into 51 subclasses at $\mathrm{L}_{2}$. As a result, 6 prediction models are constructed to predict 51 subclasses at $\mathrm{L}_{2}$. Similarly, due to the increasing number of subclasses at each subsequent level, 51 and 169 models are constructed to predict 169 and 1,921 subclasses at $\mathrm{L}_{3}$ and $\mathrm{L}_{4}$, respectively (Table 3). This hierarchical design of prediction models ensures that subclasses of a superclass are not predicted outside of that superclass and hence minimize false positives. For instance, members of EC 1.x.x.x superclass are never predicted as members of EC 2.x.x.x, and so on and so forth.

\section{Evaluation of prediction performance}

All feature vectors were randomly divided into 80 and 20 percent subsets for training and testing, respectively. Since the datasets are unbalanced across classes (and subclasses), class distributions are approximately preserved at all EC levels using stratified partitioning for training and testing sets. We used a two-step validation procedure that include determining a10-fold cross-validation accuracy on the training set, and the testing accuracy using the testing dataset that is not a part of the training data. We also report standard performance measures over each class at each level, including true positive rate (TPR), false positive rate (FPR), and receiver operating characteristic (ROC) curves and the area under the curve (AUC). Please refer to the Methods section for more details.

Table 4 shows the 10-fold accuracy and the testing accuracy for each of the five ML algorithms (Additional file 1: Figure S1). Overall, these two accuracies are consistent across the five classifiers indicating that models are not over trained. With the exception of decision stump (DS) classifier, the other four classifiers achieved at least $92.5 \%$ and $95.9 \%$ testing accuracies at $\mathrm{L}_{0}$ and $\mathrm{L}_{1}$, respectively. The three top performing classifiers are KNN, SVM and RFC, where testing accuracies reached at least $94.4 \%$ and $97.3 \%$ at $\mathrm{L}_{0}$ and $\mathrm{L}_{1}$, respectively. At the same time the false positive rates are very low; at LO
Table 4. Ten-fold cross validation and testing accuracy for enzyme identification and enzyme classification

\begin{tabular}{lcccc}
\hline & \multicolumn{2}{c}{$\begin{array}{c}\text { Enzyme Identification } \\
\left(E C L_{\mathbf{0}}\right)\end{array}$} & \multicolumn{2}{c}{$\begin{array}{c}\text { Enzyme Classification } \\
\left(E C \mathrm{~L}_{\mathbf{1}}\right)\end{array}$} \\
\hline Classifiers & $\begin{array}{c}\text { Ten-fold } \\
\text { Accuracy* }\end{array}$ & $\begin{array}{c}\text { Testing } \\
\text { Accuracy }\end{array}$ & $\begin{array}{c}\text { Ten-fold } \\
\text { Accuracy* }\end{array}$ & $\begin{array}{c}\text { Testing } \\
\text { Accuracy }\end{array}$ \\
\hline DS & 66.39 & 66.39 & 39.12 & 39.31 \\
NBC & 92.60 & 92.46 & 96.11 & 95.88 \\
KNN & 94.38 & 94.38 & 97.80 & 97.56 \\
SVM & 95.69 & 94.86 & 98.34 & 98.39 \\
RFC & 98.42 & 94.60 & 97.50 & 97.28 \\
\hline
\end{tabular}

*Ten-fold cross validation accuracy. At EC L0 and EC L1 using ML classifiers, Decision Stump (DS), Naïve Bayes Classifier (NBC), K-Nearest Neighbor (KNN), Support Vector Machine (SVM), and Random Forest Classifier (RFC). At EC L0, train and test sets contain 154,592 and 38,648 sequences respectively, whereas EC L1 contain train and test sets of 50,139 and 12,535, respectively.

and L1, the FPR for the top three methods ranges from 0.054 to 0.061 and 0.011 to 0.026 , respectively.

Figure 2 illustrates ROC curves that show the relationship between TPR (sensitivity) and FPR (1-specificity) for a single class. An ideal ROC curve heads straight up on the Y-axis and then to the right parallel to the $\mathrm{X}$-axis of the graph; thus maximizing the area under the curve (AUC). Such curves indicate that the classifier is predicting maximum true positives with minimum false positives, with AUC values closer to one. Figure 2 shows fairly consistent ROC curves for the top performing three $M L$ methods at $\mathrm{L}_{0}$ and $\mathrm{L}_{1}$, respectively. At $\mathrm{L}_{1}$, enzymes from EC6 class are consistently the best predicted, while those from $\mathrm{EC} 2$ class showed relatively the least performance. This is probably because EC2 is one of the largest classes with most divergent subclass distribution, while EC6 has the least number of subclasses. The minimum AUC values at $L_{0}$ and $L_{1}$ are 0.945 and 0.989 , respectively, indicating the superior performance of these three classifiers; hence, we chose only these three classifiers (KNN, SVM, RFC) for further use in this study.

\section{Consensus-based ensemble approach}

A consensus approach adds confidence to the prediction accuracy and drastically reduces the false positives; hence, we implemented it by considering only those predictions, where the same enzyme class is predicted for a

Table 3. Overall prediction accuracy of ECemble method

\begin{tabular}{lcccccc}
\hline EC Level & $\begin{array}{c}\text { Number of } \\
\text { Sequences }\end{array}$ & $\begin{array}{c}\text { Correctly } \\
\text { Predicted* }\end{array}$ & $\begin{array}{c}\text { \% Overall } \\
\text { Accuracy }\end{array}$ & Classes & Model(s) & Average \# of features per model \\
\hline Level-0 & 193240 & 188866 & 97.74 & 2 & 1 & 14241 \\
(Enzymes) & $(64948)$ & $(62674)$ & $(96.50)$ & & 1 & 5045 \\
Level-1 & 62674 & 62167 & 99.19 & 6 & 6 & 811 \\
Level-2 & 62167 & 61721 & 99.28 & 51 & 51 & 95 \\
Level-3 & 61721 & 60931 & 98.72 & 169 & 169 & 30 \\
Level-4 & 60931 & 60199 & 98.80 & 1921 & & \\
\hline
\end{tabular}

${ }^{*}$ Correctly predicted: Instances predicted by at least 2 of top 3 classifiers. Level-0 represents the model to predict if a protein sequence is an enzyme or not. Level 1-Level 4 represents corresponding levels of the EC number hierarchy. 


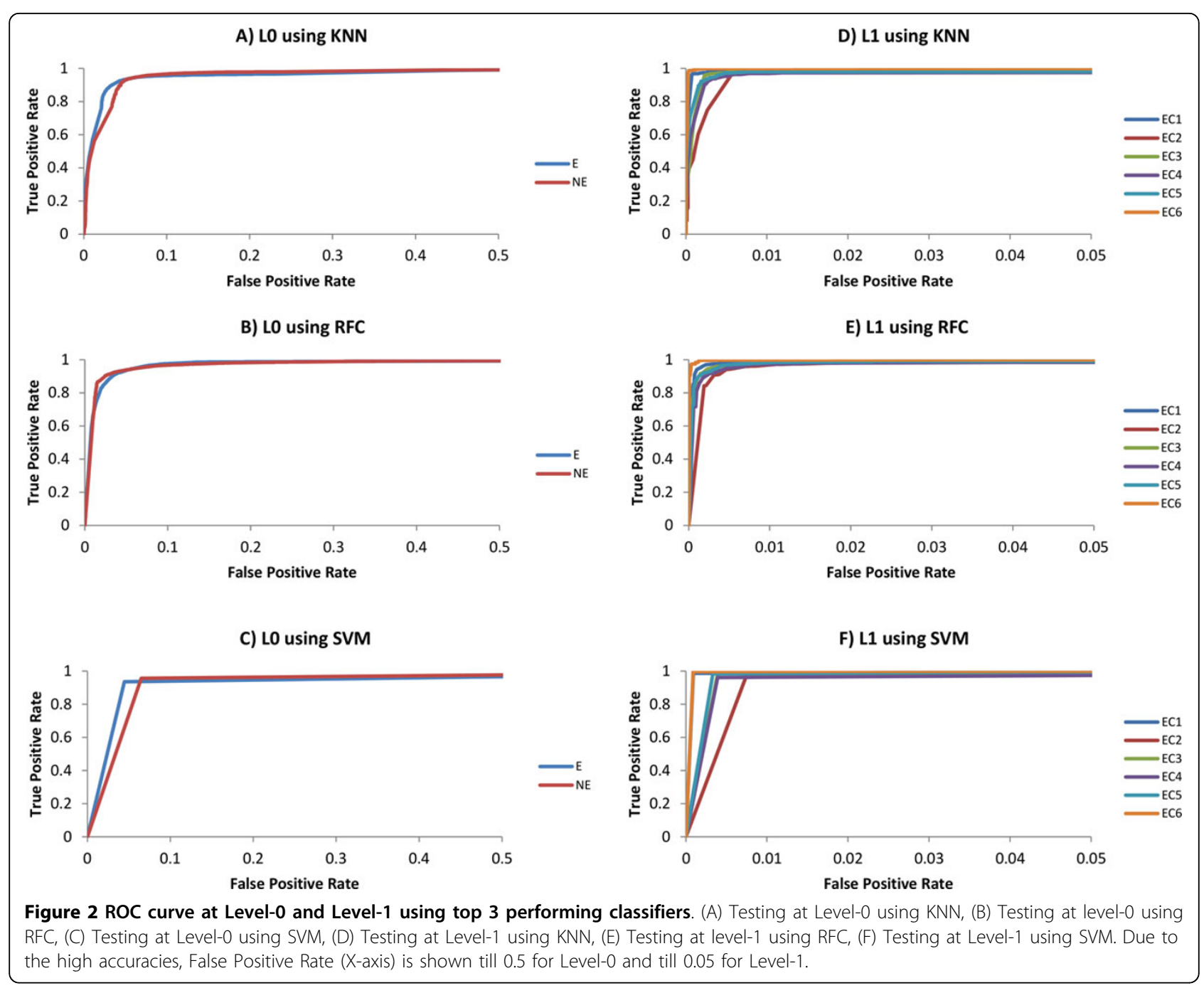

sequence by at least two out of the top three classifiers. Table 3 shows the overall prediction accuracy for testing data at each level $\left(\mathrm{L}_{0}-\mathrm{L}_{4}\right)$ after using the consensus approach. We achieved an overall accuracy of $97.7 \%$ at $\mathrm{L}_{0}$ for identifying enzymes and non-enzymes, and at least $98.7 \%$ accuracy using models at $\mathrm{L}_{1}$ to $\mathrm{L}_{4}$ for predicting enzyme classes and subclasses. These results are very promising despite the fact that the size of the training data per model kept diminishing as the number of models increase at the lower levels $\left(\mathrm{L}_{2}-\mathrm{L}_{4}\right)$. It can also be noted that at $\mathrm{L}_{0}, 96.7 \%$ of the correctly predicted instances are consistent across the top three classifiers, while at $\mathrm{L}_{1}-\mathrm{L}_{4}$, over $99 \%$ of the correctly predicted instances are consistent (Additional file 2: Table S1).

\section{Effect of sequence identity in the training dataset}

Sequence redundancy in the training datasets often results in overtraining and inaccurate estimation of prediction accuracy. To test the effect of sequence identity on the prediction accuracy, we created four datasets at $70 \%, 60 \%$, $50 \%$ and $40 \%$ sequence identities using the CD-HIT clustering algorithm [43] and accordingly labeled as cdh70, cdh60, cdh50, and cdh40. At lower sequence identity thresholds like $40 \%$, more sequences got removed resulting in a fewer number of sequences in each enzyme class compared to datasets with higher sequence identities. Similarly, the number of enzyme classes containing minimum number of sequences (ten) for model building started to go down from thresholds 70 to 40 percent identity (Additional file 3: Figure S2A). We generated the enzyme prediction models for EC levels $\mathrm{L}_{0}-\mathrm{L}_{4}$ for all the 4 datasets (shown in Additional file 4: Table S2). We needed at least 10 sequences in each subclass to build models using 10-fold cross validation. Accuracy is the highest for the cdh70 dataset ( $70 \%$ sequence identity) compared to all other datasets (cdh60, cdh50, cdh40) (Additional file 3: Figure S2B); hence, we used this dataset for model building. The $70 \%$ sequence identity is 
considered an optimal threshold in many other ML datasets, because the enzyme function starts to diverge quickly when the sequence identity is below 70\% [5].

\section{Comparison of ECemble with other methods}

We compared the performance of ECemble with that of two existing methods, BLAST and EFICAz [24]. We chose these two methods because a number of methods are BLAST-based homology searching methods and EFICAz is a popular open-source tool. We used the same train and test datasets against these three methods to compare their performance. In the first step, a BLAST database was created with train dataset and the test data was queried against it for enzyme identification. Because BLAST generates a number of hits for each query with varying levels of confidence, only the top hit was considered (with a minimum E-value threshold of $10^{-5}$ for blastp) as the prediction for each sequence in the test set. To predict the enzyme classes and subclasses, we performed a second query against a BLAST database created using only the enzyme sequences and used the same procedure as in the first step. Similarly, EFICAz method was trained and tested for both enzyme identification and classification. We also used four different clustered datasets (using CD-HIT) that were described earlier. For enzyme identification, ECemble reports the highest accuracy (94.9\%) compared to BLAST (89\%) and EFICAz (88.9\%) using cdh70 dataset (Figure 3A). It can also be seen that the accuracy goes down as the percent identity in the datasets goes down from 70 to 40 ; however, this effect is more pronounced in the BLAST method compared to ECemble and EFICAz suggesting that reduced identity has minimal effect on these two methods. Similarly for enzyme classification at $\mathrm{L}_{1}$, the overall accuracy of ECemble (98.4\%) is better than BLAST (28.43\%) and EFICAz (93.36\%) methods using cdh70 dataset (Figure 3B). We compared four different sequence identity thresholds $(70 \%, 60 \%, 50 \%$ and $40 \%$ ) of this dataset and our method performed better than others irrespective of the dataset used. These results convincingly demonstrate that the ECemble method consistently performed better than BLAST and EFICAz methods at all identity thresholds $(70 \%, 60 \%, 50 \%, 40 \%)$, and that it is also suitable for accurate annotation of protein sequences with low sequence identity. Hence, in the next section, we used ECemble to identify and annotate complete enzyme complements of the sequenced genomes and metagenomes and applied this method to study the role of gut microbiome-derived enzymes in human metabolism.

\section{Application of ECemble method}

\section{Annotation of full complements of enzymes in} sequenced genomes

The ability of ECemble to predict solely based on a protein sequence enables it to annotate full complements of enzymes from sequenced genomes as well as from mixed genomes such as metagenomic samples. We applied ECemble to annotate a large number of unknown or unclassified enzyme sequences from 10 proteomes (that include reviewed and unreviewed proteins from UniProt database [44]) of both eukaryotic and prokaryotic model organisms (Additional file 5). As seen in Figure 4, E. coli and yeast proteomes have a high fraction of known enzymes $(27 \%$ and $24 \%$, respectively) compared to less than $5 \%$ in most of the other species. In contrast, the fractions of ECemble predicted enzymes are smaller in E. coli and yeast (around 4.5\%), but reach up to $12 \%$ in chicken and zebrafish proteomes. In human and mouse, the fractions of unknown or unclassified enzymes predicted by ECemble account to $7.4 \%$ and $8.1 \%$ of their proteomes, respectively. These data underscore an important and generic application of our method that is to annotate a large number of unknown enzymes and enzyme classes in the sequenced genomes.

We further investigated about 200 newly predicted enzymes from the reviewed human proteome sequences (from the SwissProt database [45]), that were either not known as enzymes or had partially annotated enzyme subclasses. These 200 enzymes map to 119 unique enzyme reactions (with unique EC numbers), of which, only 30 are not previously known to be in human. Table 5 lists these 30 enzymes with the current annotation in UniProt and the predicted annotation by ECemble. Note that despite the well-characterized nature of the reviewed proteins, ECemble method is able to complement or correct the existing enzyme annotations (marked with * in Table 5). For instance, human genes such as FOXRED1 (FAD-dependent oxidoreductase domain-containing protein 1) and SCCPDH (saccharopine dehydrogenase-like oxidoreductase) have been broadly known as oxidoreductases and accordingly labeled as 1.-...-, in the UniProt database. ECemble method predicted the specific reactions of these enzymes as malate dehydrogenase (quinone) [EC 1.1.5.4] and Saccharopine dehydrogenase $(\operatorname{NADP}(+)$, L-glutamate-forming) [EC 1.5.1.10], respectively. In certain cases (marked with ** in Table 5), ECemble predictions differ only at the subclass levels $\left(\mathrm{L}_{2}-\mathrm{L}_{4}\right)$, while in a small number of cases (marked with ${ }^{* * *}$ in Table 5), the predictions also differ from the current annotations at the class level $\left(\mathrm{L}_{1}\right)$. Hence, experimental validation of these predictions is worth pursuing in the future. Because ECemble prediction models are built only from reviewed enzyme sequences, the accuracy and coverage of prediction by our model will continue to improve as the newly characterized enzymes from experimental studies become available. These results prove that ECemble method that solely uses protein sequences for prediction, 


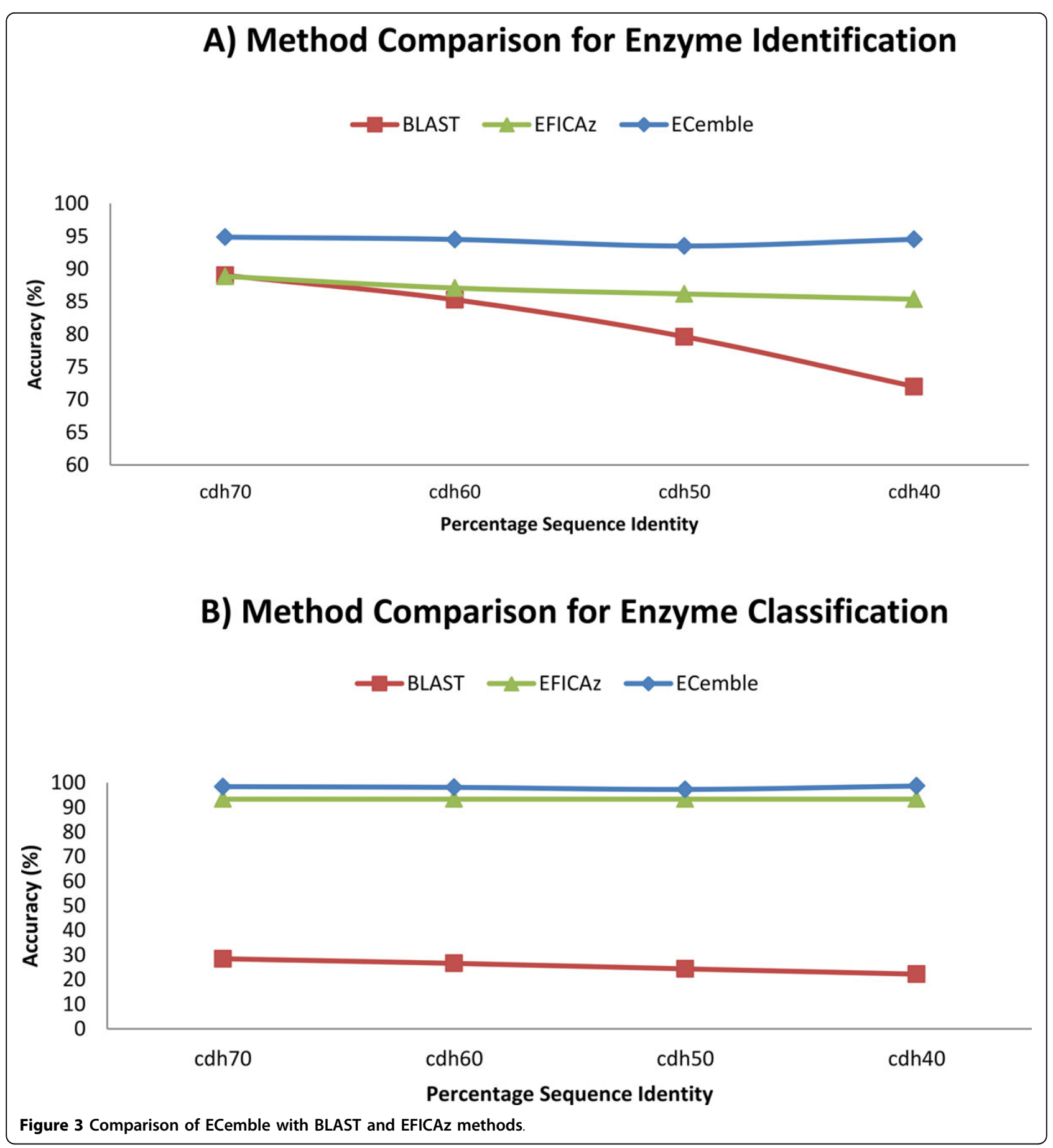

is highly promising for the identification and classification of full complements of enzymes in the sequenced genomes.

\section{Prediction of enzymes from the gut microbiome}

To understand the role played by the most densely colonized human microbiome (gut microbiome) in human metabolism, we would require a catalogue of all the enzymes that can potentially exist in the human gut environment. There are several hundreds to thousands of microbial species that inhabit human gut and many of them are unknown, under characterized or not culturable in laboratory conditions, which would have made our goal impossible to accomplish. However, Next-Generation Sequencing (NGS) data from metagenomic samples could be used for de novo assembly of 


\section{Known and ECemble Predicted Enzymes in Uniprot}

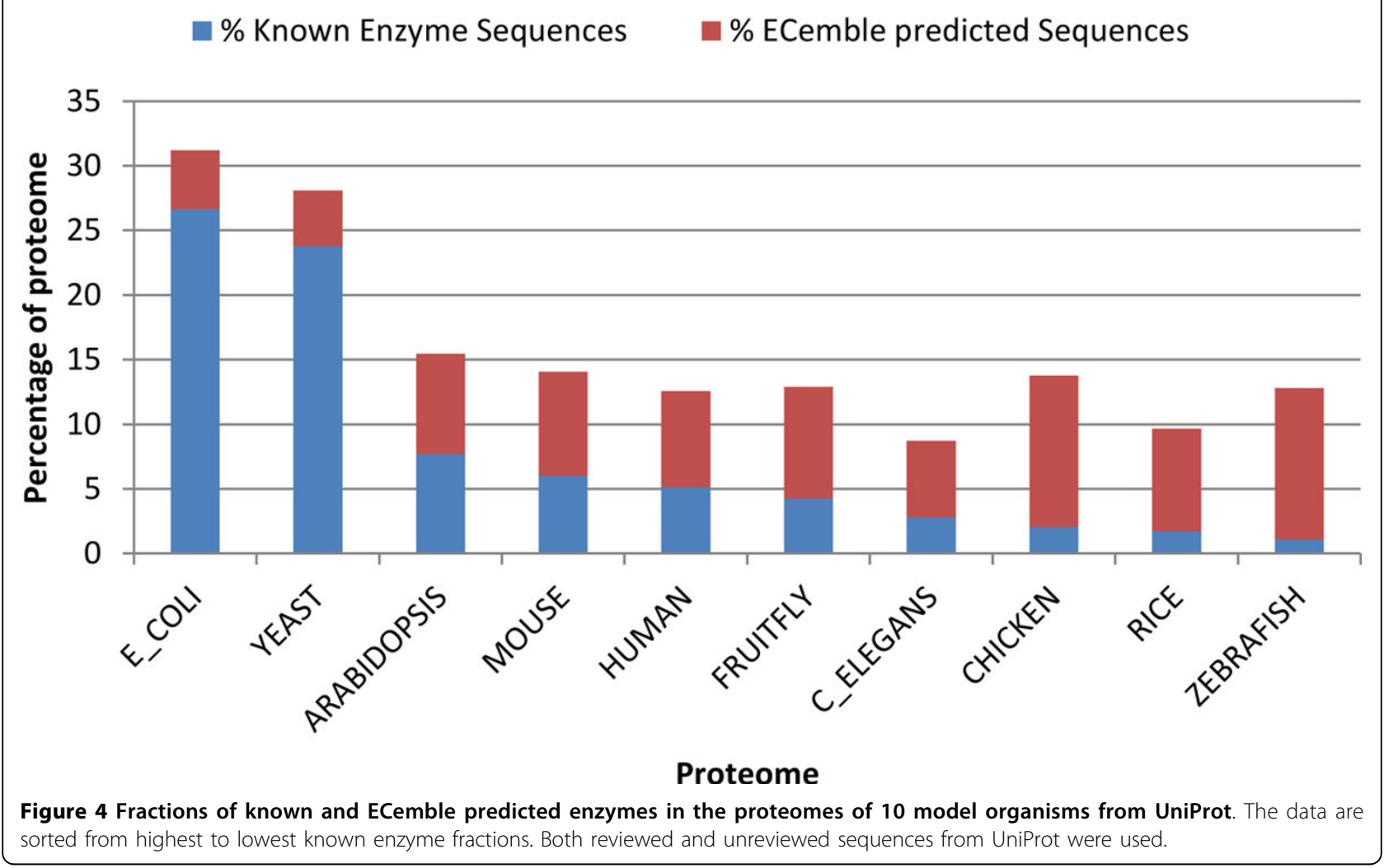

microbial genomes, and consequently for the prediction of the translated proteomes from the assembled scaffolds. We applied the ECemble method on 2.5 million proteins from metagenomic sequences (discussed in the Methods section) and assigned 213,313 (8.53\%) sequences to 513 distinct enzymatic reactions (Additional file 6). Of these, 276 reactions are also encoded in human, leaving 237 reactions that are exclusively encoded by human gut microbial genomes. Of these, 222 enzymes are known gut bacterial enzymes in SwissProt database, while the remaining 15 enzymes are newly predicted by the ECemble method.

\section{Role of gut microbial enzymes on human metabolism} Application of ECemble to identify the enzymes and enzyme classes in human and gut microbial species has enabled us to ask the following questions. Which gut microbial enzymes are involved in human metabolism? Which human pathways are partly or fully driven by microbe-derived enzymes? Are there any previously unknown microbial enzymes that are involved in human gut metabolism? Even though, gut microbiome can contains bacteria, fungi and other small eukaryotes, about $98 \%$ of the gut microbial enzymes used in this study originated from bacteria [26], hence we limited this study only to bacteria-derived enzymes. To answer these questions, we created 4 different sets of enzymes. (i) Known human enzyme reactions (with unique EC numbers) (1,271), (ii) Unknown human enzyme reactions that are predicted by ECemble (19), (iii) Known bacterial enzyme reactions in the gut microflora predicted by ECemble (222) and (iv) Unknown gut bacterial enzyme reactions that are predicted by ECemble (15). For human and bacteria, known enzymes were obtained from SwissProt and KEGG (Kyoto Encyclopedia of Genes and Genomes) [46] databases (Additional file 7 shows known human enzyme reactions).

We mapped both human- and gut bacteria-encoded enzymes to the KEGG reference pathways to identify 48 human metabolic pathways, where each pathway contains both human- and bacteria-encoded enzymes plus at least one of them is predicted by ECemble. We refer to them henceforth as gut microbe complemented (GMC) pathways. The first set (39 pathways) contains human enzymes that are complemented by known gut bacterial enzymes. This set serves to validate the known role of gut bacteria in human metabolism (Additional file 8: Figure S3). The second set (9 pathways) is same 
Table 5. ECemble predicted enzymes from reviewed human proteome

\begin{tabular}{|c|c|c|c|c|}
\hline Gene Name & Accession & \$UniProt Annotation & New ECemble Prediction & $\begin{array}{l}\text { EC Description of } \\
\text { Enzyme Function }\end{array}$ \\
\hline DHRS7 & Q9Y394 & 1.1.-- & 1.1.1.n $4^{*}$ & (-)-trans-carveol dehydrogenase \\
\hline GFOD1 & Q9NXC2 & $1 .---$ & 1.1.1.n6* & D-chiro-inositol 3-dehydrogenase \\
\hline FOXRED1 & Q96CU9 & $1,-\cdots$ & 1.1.5.4* & Malate dehydrogenase (quinone) \\
\hline$A L O X 12 B$ & O75342 & 1.13.11.- & 1.13.11.12* & Linoleate 13S-lipoxygenase \\
\hline ALDH8A1 & Q9H2A2 & 1.2.1.- & 1.2.1.16* & Succinate-semialdehyde dehydrogenase (NAD(P)(+)) \\
\hline SCCPDH & Q8NBX0 & $1 .--\cdot$ & $1.5 .1 .10^{*}$ & Saccharopine dehydrogenase (NADP(+), L-glutamate-forming) \\
\hline TFB1M & Q9UNQ2 & 2.1.1.- & 2.1.1.182* & 16S rRNA (adenine(1518)-N(6)/adenine(1519)-N(6))-dimethyltransferase \\
\hline METTL15 & A6NJ78 & 2.1.1.- & 2.1.1.199* & 16S rRNA (cytosine(1402)-N(4))-methyltransferase \\
\hline METTL15P1 & P0C7V9 & 2.1.1.- & 2.1.1.199* & 16S rRNA (cytosine(1402)-N(4))-methyltransferase \\
\hline PYROXD2 & Q8N2H3 & $1 .--\cdot$ & $2.1 \cdot 1.74^{*}$ & $($ FADH(2)-oxidizing $)$ \\
\hline GOT1L1 & Q8NHS2 & 2.6.1.- & 2.6.1.9* & Histidinol-phosphate transaminase \\
\hline FGGY & Q96C11 & 2.7.1.- & 2.7.1.16* & Ribulokinase \\
\hline ISPD & A4D126 & 2.7.7.- & 2.7.7.60* & 2-C-methyl-D-erythritol 4-phosphate cytidylyltransferase \\
\hline GDPD3 & Q7L5L3 & 3.1.-- & $3.1 .4 .46^{*}$ & Glycerophosphodiester phosphodiesterase \\
\hline TLL1 & O43897 & 3.4.24.- & $3.4 .24 .21^{*}$ & Astacin \\
\hline TASP1 & Q9H6P5 & 3.4.25.- & 3.4.25.2* & HsIU-HsIV peptidase \\
\hline$A D A L$ & Q6DHV7 & 3.5.4.- & $3.5 .4 .2^{*}$ & Adenine deaminase \\
\hline IREB2 & P48200 & None & 4.2.1.33* & 3-isopropylmalate dehydratase \\
\hline TYRP1 & P17643 & 1.14.18.- & 1.14.11.13** & Gibberellin 2-beta-dioxygenase \\
\hline HSD11B2 & P80365 & 1.1.1.- & 1.3.1.9** & Enoyl-[acyl-carrier-protein] reductase (NADH) \\
\hline NDOR1 & Q9UHB4 & 1.6.-- & $1.8 .1 .2^{* *}$ & Sulfite reductase $(\mathrm{NADPH})$ \\
\hline ENDOV & Q8N8Q3 & 3.1.26.- & $3.1 \cdot 21.7^{* *}$ & Deoxyribonuclease V \\
\hline ENPP5 & Q9UJA9 & 3.1.-- & $3 \cdot 6 \cdot 1 \cdot 27^{* *}$ & Undecaprenyl-diphosphate phosphatase \\
\hline MPPED2 & Q15777 & 3.1.-- & 3.6.1.41** & Bis(5'-nucleosyl)-tetraphosphatase (symmetrical) \\
\hline THNSL2 & Q86YJ6 & 4.2.3.- & 4.2.1.20** & Tryptophan synthase \\
\hline ALOXE3 & Q9BYJ1 & 5.4.4.- & $1.13 .11 .12^{* * *}$ & Linoleate 13S-lipoxygenase \\
\hline ABHD2 & P08910 & 3.1.1.- & $2.3 \cdot 1.31^{* * *}$ & Homoserine O-acetyltransferase \\
\hline$A B H D 1$ & Q96SE0 & 3.1.1.- & $2 \cdot 3 \cdot 1.31^{* * *}$ & Homoserine O-acetyltransferase \\
\hline ABHD3 & Q8WU67 & 3.1.1.- & $2.3 \cdot 1.84^{* * *}$ & Alcohol O-acetyltransferase \\
\hline SDR42E1 & Q8WUS8 & 1.1.1.- & $5.1 .3 .20^{* * *}$ & ADP-glyceromanno-heptose 6-epimerase \\
\hline
\end{tabular}

${ }^{\$}$ Current annotation is based on UniProt database

$n$ Preliminary EC numbers include an ' $\mathrm{n}$ ' as part of the fourth digit in ENZYME database

* Good predictions that complement the current annotations

** Predictions that differ from the current annotations only at the subclass level

*** Predictions that differ from the current annotations at the class level

as the first one; in addition, contains predicted enzymes (previously unknown) from gut bacterial species. These nine GMC pathways reveal the role of newly discovered gut bacterial enzymes in human gut metabolism, which is made possible with the ECemble method (Additional file 9: Figure S4). All the 48 pathways are mapped with enzymes (EC numbers) using a color-coded format. Light red colored enzymes represent known human enzymes, pink represents unknown human enzymes that are predicted by ECemble, light green represents known bacterial enzyme reactions in the gut microflora predicted by ECemble and light blue represents unknown gut bacterial enzyme reactions that are predicted by ECemble.

Functional distribution of GMC pathways is shown in Figure $5 \mathrm{~A}$ and a full list of 48 pathways is given in
Additional file 10. GMC pathways contribute to the metabolism of a variety of nutrients that primarily include carbohydrates, amino acids, vitamins and cofactors. Thus, the metabolic role of GMC pathways closely matches with the known nutritional requirements of humans. Our results show that gut microbial enzymes also play a role in lipid and energy metabolism and also in the metabolism of terpenoids, polyketides and derived amino acids such as taurine, D-glutamine, etc. Our method predicted 27 Carbohydrate active enzymes (CAZymes) (Additional file 11) in the human gut microbiome that include carbohydrate esterases, glycoside hydrolases, glycosyl transferases and polysaccharide lyases, which are primarily involved in carbohydrate metabolic pathways. As shown in Figure 5B, human- and 


\title{
A) Distribution of Mapped Pathways in each Pathway Category
}

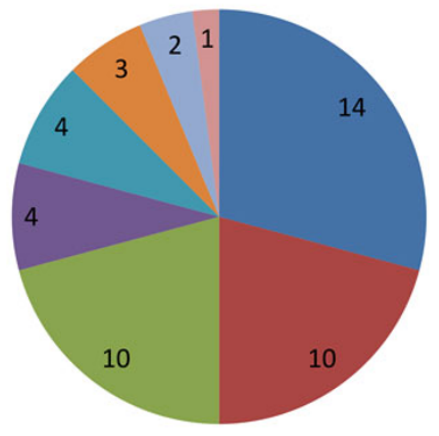

\author{
- Carbohydrate \\ Amino acid \\ - Cofactors and vitamins \\ Energy \\ = Other amino acids \\ - Lipid \\ Nucleotide \\ - Terpenoids and polyketides
}

\section{B) Distribution of Enzymes in each Pathway Category}

\author{
- Human Enzymes Bacterial Enzymes
}

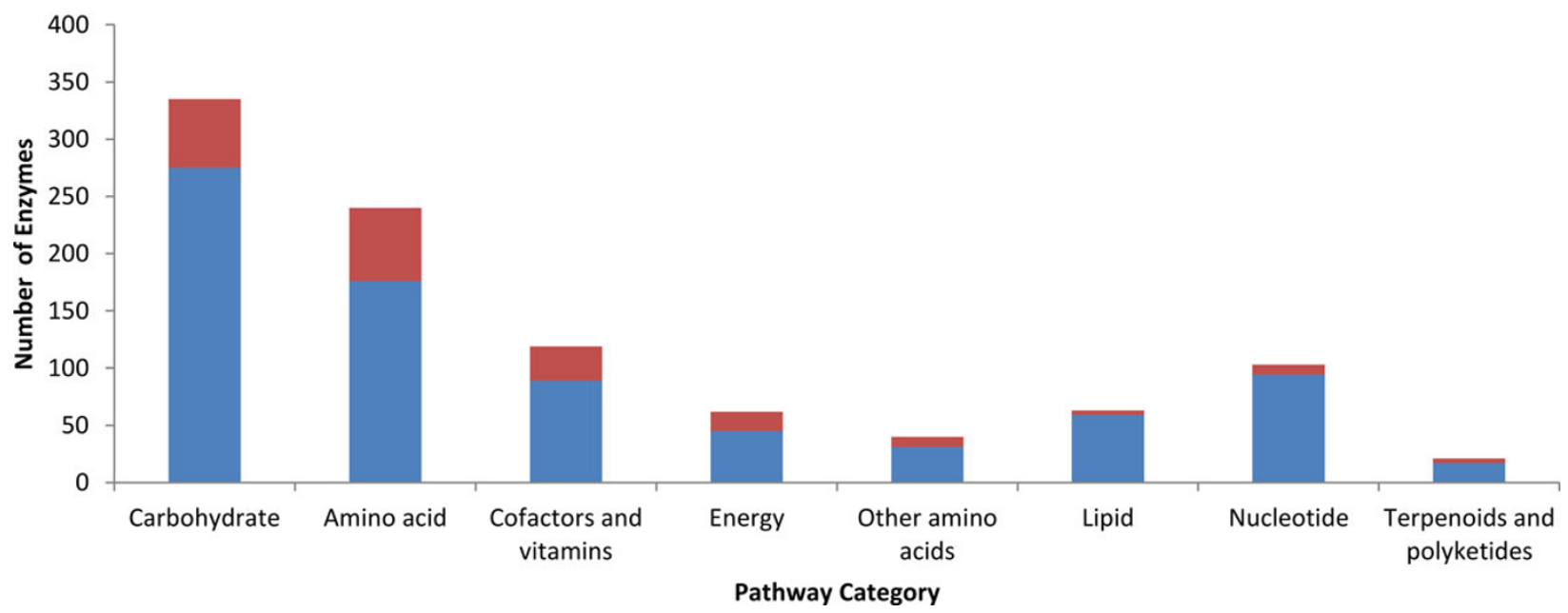

Figure 5 Distribution of pathways and enzymes in each of the major pathway categories. (A) Distribution of mapped pathways in each of the 8 major pathway categories (B) Distribution of human and bacterial enzymes in each of the 8 major pathway categories.

microbe-derived enzymes complement different categories of human metabolic pathways. Note that 64,60 and 30 exclusively microbe-derived enzymes complement the amino acid, carbohydrate and vitamin/cofactor metabolic pathways, respectively, which underscores their pivotal role as well as the dependency of human pathways on microbe-derived enzymes. Microbes not only complement, but also supplement some of the enzyme functions that are common to both human and microbes. For instance, we found that 518 enzymatic reactions are shared between human and gut-bacterial species; and we hypothesize that some of these common enzymes that are secreted by bacteria could supplement to perform human metabolic functions, and vice-versa. These common enzymes are highlighted in blue color in the Additional file 12 . These results empirically support the argument that symbiotic gut microbiome has coevolved with human (or the coelomate animals) and they play a huge role in the metabolic interactions between host-gut microbiota [32].

Humans lack the enzymes needed to degrade oxalate [47]. It has been shown that the bacterial degradation of oxalate is carried by Oxalobacter formigenes in the human intestinal tract and the absence of Oxalobacter formigenes is considered a risk factor for urolithiasis [48]. Similarly Choline, an essential dietary nutrient, is found to be metabolized in the liver by the gut microbial enzymes. Thus conversion of dietary choline is used as a metabolic hallmark for liver and cardiovascular diseases [49]. Similarly, nitrate reductase that converts nitrate into nitrite and nitric oxide, is synthesized only by gut microbiome; elevated levels of nitric oxide have been associated 
with both IBD and obesity-induced insulin resistance [31]. Gut microbiome also plays a crucial role in the metabolism of xenobiotics; at least thirty commercially available drugs are shown to be metabolized as substrates by bacterial enzymes $[50,51]$. On the other hand, gut microbiome is also a source of inflammatory molecules such as lipopolysaccharide and peptidoglycan that may contribute to metabolic diseases [52].

\section{Gut microbe-complemented pathways for dietary carbohydrate metabolism}

Carbohydrates are a major component of the human diet that includes starch (amylose and amylopectin) and disaccharides such as sucrose, lactose, and maltose. Human gut bacteria produce a vast panel of CAZymes to degrade components of dietary fiber into metabolisable monosaccharides and disaccharides [33]. The human genome encodes at best 17 digestive enzymes [53]; for ex. lactase, $\alpha$-amylase, maltase, isomaltase and sucrose. It has been known that human enzymes can hydrolyze disaccharides (sucrose, lactose and maltose, etc.) and starch, but not other complex polysaccharides [54]. Hence, our ability to digest dietary plant carbohydrates resides entirely in our gut, where gut microbe-derived enzymes can hydrolyze complex dietary carbohydrates by producing a variety of CAZymes [55]. Thirteen gut bacterial enzymes predicted by ECemble were mapped in starch and sucrose metabolism pathway as shown in Figure 6. Enzymes responsible for the conversion of sucrose to glucose and bacterial degradation of pectin (a common component of dietary fibers) and xylan (polysaccharides in plant cell walls) are shown in the Table 6 . These enzymes are predicted by our ECemble method from the gut microbial metagenomic data.

Another GMC pathway, fructose and mannose metabolism, explains how bacterial enzymes complement human enzymes to metabolize dietary sugars (Figure 7). Fructose occurs as a free monosaccharide and an isomer of glucose. In Figure 7, predicted bacteria-encoded enzymes and known human-encoded enzymes are shown, where D-Fructose (fructose) is catalyzed by bacterial enzymes, Protein-N (pi) -phosphohistidine-sugar phosphotransferase (2.7.1.69) and Fructokinase (2.7.1.4) into D-Fructose-1 Phosphate and $\beta$-D-Fructose- 6 Phosphate, respectively. $\beta$-D-Fructose-6P is metabolized to Glyceraldehyde-3P using human-encoded enzymes Phosphofructokinase (2.7.1.11) and Fructose-biphosphate aldolase (4.1.2.13). The Glyceraldehyde-3P compound is a part of the glycolysis (normal metabolism of sugars) pathway, which is the main energy generating mechanism in the body. This pathway demonstrates that the bacteria- and humanencoded enzymes complement and work in unison in the digestion and energy metabolism pathways.

\section{Analysis of enzyme profiles of obese and IBD subjects}

A recent study published the metagenomic profiles of obese, lean and Inflammatory Bowel Disease (IBD) subjects [26]. This study also reported the translated gene products of gut microbiome from 124 metagenomic subject samples. We used these protein sequences to predict all the enzymes in each subject with our ECemble method, and analyzed the enzyme profiles of 'obese versus lean' (42 obese/82 lean) and 'IBD versus nonIBD' (25 IBD/99 Non-IBD) subjects. We identified 237 unique bacterial enzymes that are not encoded in human from the metagenomic samples of obesity, lean, IBD and non-IBD subjects. These include 222 known and 15 previously unknown enzymes in gut bacterial species. Details on how these enzyme reactions maps to KEGG human pathways are shown in Additional files 13 and 14. The taxonomic distribution of bacterial species from metagenomic samples is also shown in Additional file 15. The frequencies of enzymes present in the subjects were normalized based on the number of subjects in the obese/lean and IBD/non-IBD comparison groups and a Fisher's exact-test (P-value <0.05) using $\mathrm{R}$ [56] was conducted to determine the significantly enriched or depleted enzymes in the obese and IBD subjects (Additional files 16 and 17). Of the obesity-enriched enzymes, the most significant enzyme (P-value, 9.84 $\mathrm{E}^{-04}$ ) is polygalacturonase (EC 3.2.1.15), which is encoded by Bacteroides and Prevotella species, and carries out pentose and glucuronate interconversions in starch and sucrose metabolism (Additional file 16). In contrary, urease (EC: 3.5.1.5) encoding bacteria are found in fewer number of obese subjects compared to lean subjects (obese $/$ lean ratio $=0.88, \mathrm{P}$-value $=0.0179$ ), suggesting that the loss or absence of this enzyme may be associated with obesity. In our analysis of IBD bacterial enzymes (Additional file 17), we found that non-IBD subjects predominantly host bacterial populations that contain an enzyme, Glucose-1-phosphate thymidylyltransferase (EC: 2.7.7.24, IBD/Non-IBD ratio=0.06; $\mathrm{P}$-value $=2.9 \mathrm{E}^{-12}$ ) compared to the IBD group. This enzyme is involved in the biosynthesis of L-rhamnose in bacteria. While there is no direct evidence to link lower levels of L-rhamnose to IBD, increased lactulose/Lrhamnose permeability ratio or decreased L-rhamnose in human intestinal permeability is found to be associated with IBD $[57,58]$. Hence, the consequences of the absence of bacterial populations that produce L-rhamnose in the IBD patients is worth investigating by experimental studies. The distribution of significant (p-value $<0.05$ ) enzymes in obese/lean and IBD/Non-IBD groups is presented in Figure 8. The comprehensive list of enzymes in each category (obese/lean and IBD/Non-IBD) is given in Additional files 16 and 17. 


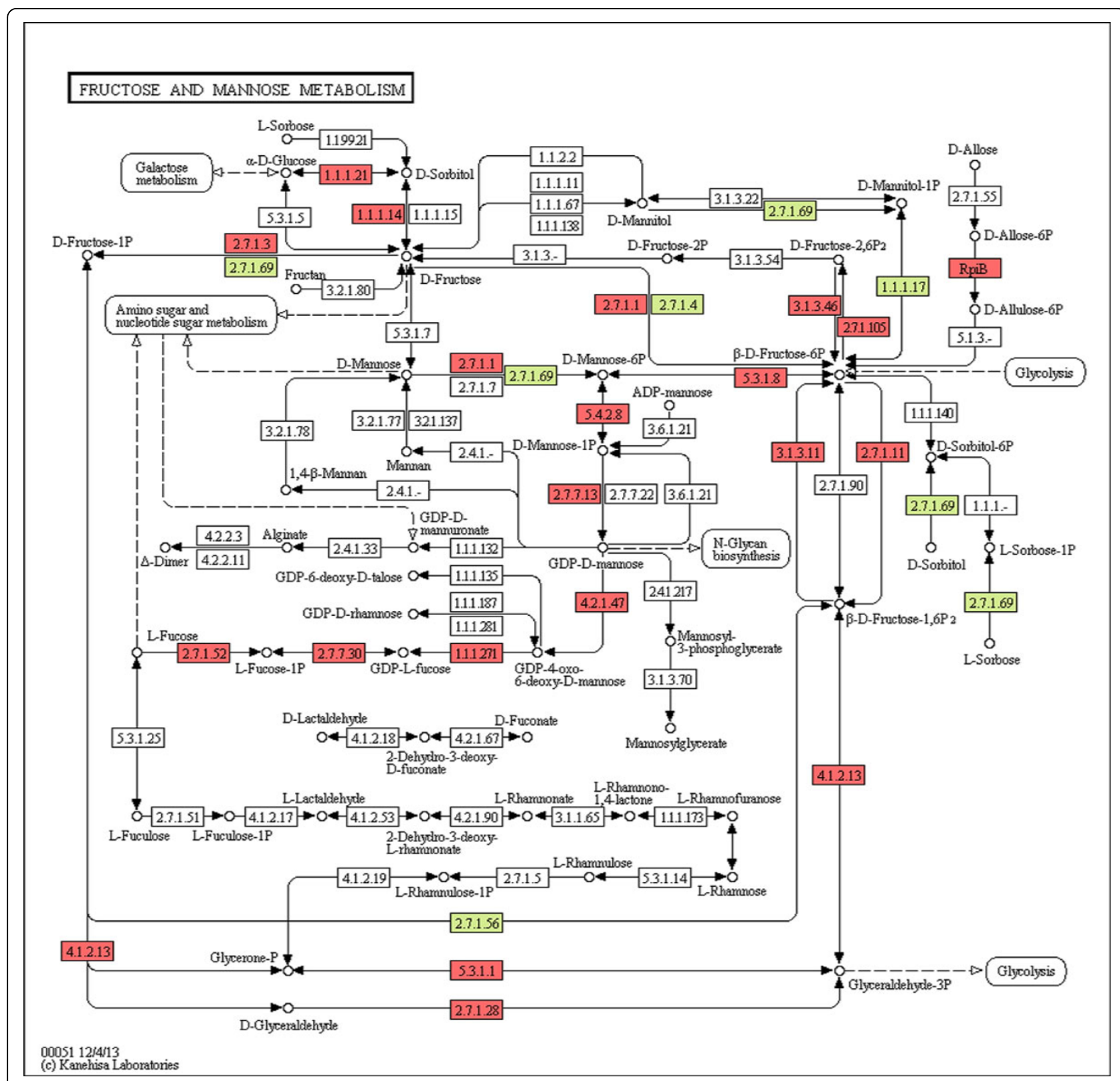

Figure 6 Starch and sucrose metabolism pathway. The following color coding scheme is used for the pathways: Known human enzyme reactions (Light Red), Unknown human enzyme reactions that are predicted by ECemble (Pink), Known bacterial enzyme reactions in the gut microflora predicted by ECemble (Light green) and Unknown gut bacterial enzyme reactions that are predicted by ECemble (Light Blue).

Table 6. Enzymes and their function in starch and sucrose metabolism pathway

\begin{tabular}{lcc}
\hline Function & Enzyme used & EC Number \\
\hline Sucrose $\rightarrow$ Sucrose-6P & Protein-N(pi)-phosphohistidine-sugar phosphotransferase & 2.7 .1 .69 \\
Sucrose-6P $\rightarrow$-D-D-Glucose-6P (glucose) & Beta-fructofuranosidase & 3.2 .1 .26 \\
Pectin $\rightarrow$ Pectate & Pectinesterase & 3.1 .1 .11 \\
Pectate $\rightarrow$ Galacturonate & Polygalacturonase & 3.2 .1 .15 \\
Xylan $\rightarrow$ Xylose & Xylan 1,4-beta-xylosidase & 3.2 .1 .37 \\
\hline
\end{tabular}




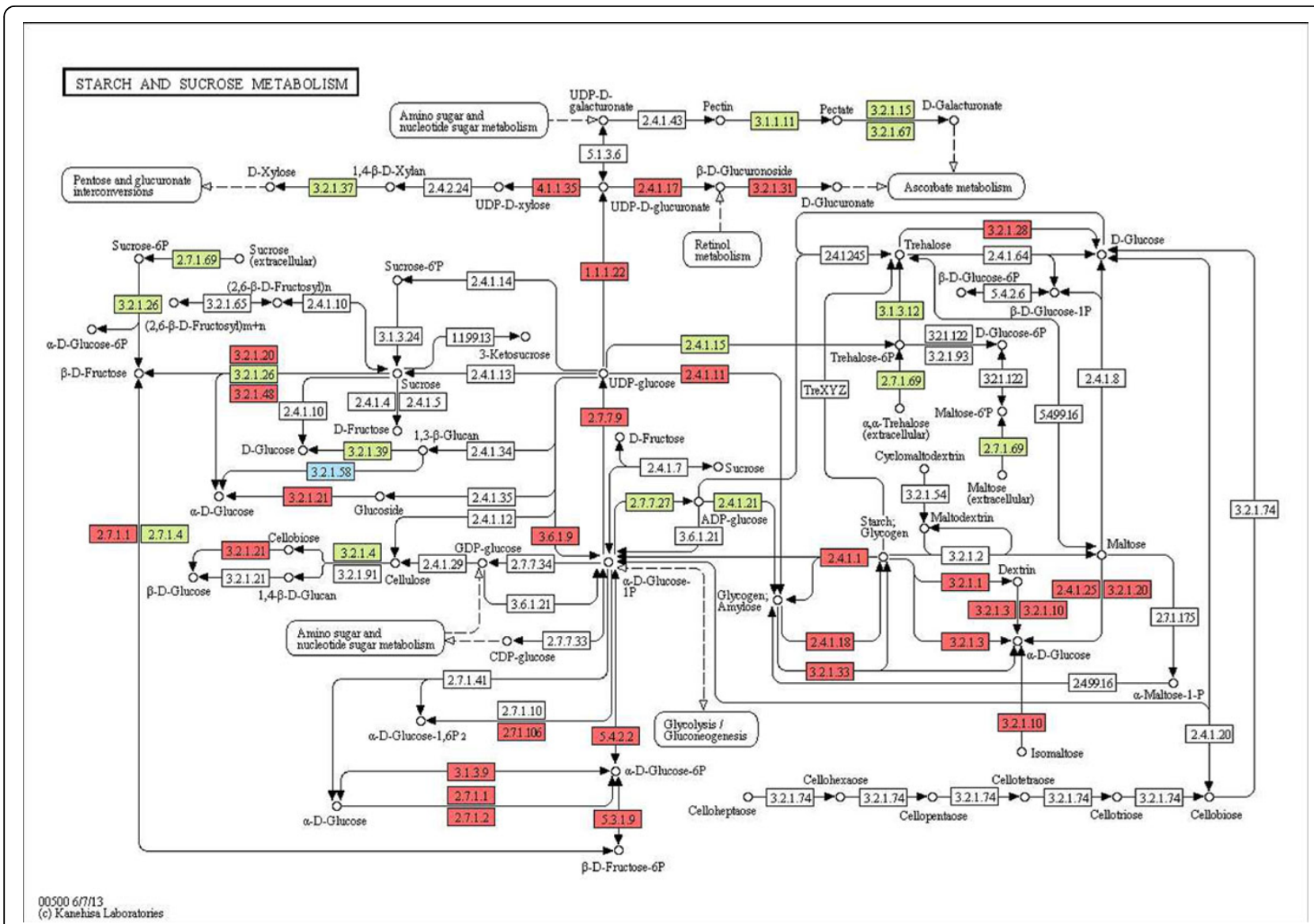

Figure 7 Fructose and mannose metabolism pathway. The following color coding scheme is used for the pathways: Known human enzyme reactions (Light Red), Unknown human enzyme reactions that are predicted by ECemble (Pink), Known bacterial enzyme reactions in the gut microflora predicted by ECemble (Light green) and Unknown gut bacterial enzyme reactions that are predicted by ECemble (Light Blue).

\section{Conclusions}

A consensus-based ensemble method, ECemble, was implemented to identify enzymes from non-enzymes, and to hierarchically predict the class and subclasses of an enzyme up to $\mathrm{L}_{4}$ of the $\mathrm{EC}$ number. Comparison against BLAST and EFICAz methods showed superior performance of ECemble, both in coverage and accuracy. The superior coverage can be attributed to the generic protein domain feature space used in this method, while the improved accuracy resulted from the stringent consensus-based ensemble approach. Application of ECemble to predict full complements of enzymes from 10 sequenced genomes of model organisms has generated new annotations for unknown enzymes as well as full annotations for undercharacterized enzymes. Similarly, ECemble method enabled us to predict bacterial enzymes present in the gut microbiome and consequently use them to study the dependence of human metabolism on gut microbe-derived enzymes. Mapping of human and microbe-derived enzymes to KEGG metabolic pathways revealed that gut microbe-derived enzymes, especially those involved in the digestion of dietary nutrients, are essential components of a number of human pathways. Further application of this method to study the profiles of gut microbe-derived enzymes in lean versus obese, and IBD versus non-IBD subjects showed that certain enzymes were significantly enriched or depleted in these comparison groups, warranting further studies to understand the role of these enzymes on certain disease conditions. Two important merits of ECemble are that it can predict solely based on the protein sequence and also fully annotates enzymes by hierarchically assigning classes and subclasses up to $\mathrm{L}_{4}$. Hence, it can be a valuable tool for accurately annotating the entire enzyme complements of individual genomes as well as the mixed genomes from metagenomic studies. As evident from this study, ECemble can be effectively used to study the metabolic interactions between the host and microbes or those among the members of a community in a microflora. Being a generic method, it can be applied to study the systems level pathway interactions in any organism or microbial community. 


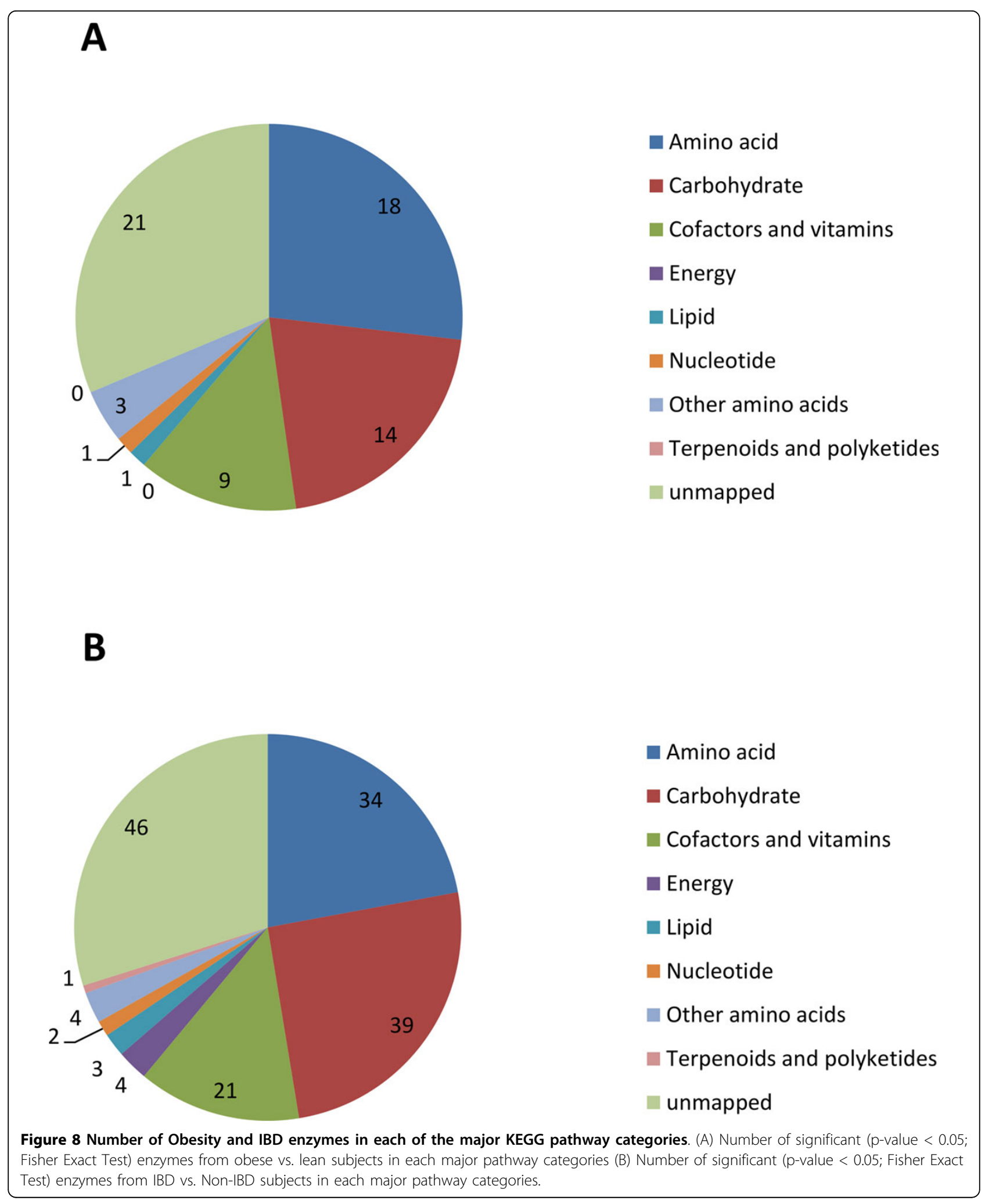




\section{Methods}

\section{ECemble dataset}

The dataset include enzyme sequences taken from the EXPASY enzyme database [59], which is built based on SwissProt database [45] that contains reviewed and experimentally determined annotations on enzymes. We applied the following filters to obtain high-quality data for testing and training our program: duplicate sequences, sequences annotated as 'fragments' and those shorter than 50 residues in length were removed; sequences having more than one EC number were also removed. To avoid redundancy in the dataset, no more than $70 \%$ sequence identity was allowed among sequences within a class for each of the six EC classes, using the CD-HIT program [43]. Consequently, 64,950 non-redundant enzyme sequences (positive dataset) were collected that broadly cover all the known enzyme classes and subclasses. Similarly, a negative dataset (non-enzymes) of 128,475 sequences were collected from a SwissProt database by following the same filtering steps as mentioned above. A class-wise distribution of known enzyme sequences is listed in Table 7. Sequence to domain mapping was done for enzyme and non-enzyme sequences based on the Pfam [38] and Superfamily [39] databases using HMMSCAN [60], and the Prosite database using PSSCAN [61].

\section{Feature databases}

The most important features of enzymes that differentiate them from non-enzymes are their structure, function and catalytic sites. The feature set we used for machine learning in this study was based on the functional and structural domains, and sequence motifs. Structural domains define evolutionarily conserved region of proteins that can fold independently, while functional domains define evolutionarily conserved regions that can independently perform a specific biological function. Similarly, short sequence motifs define the catalytic or binding sites of enzymes. Machine learning algorithms exploit these different sets of features from enzyme and non-enzyme data in differentiating one from other.
Three popular domain databases - Pfam, Superfamily and Prosite - were used to extract the domain features of enzyme and non-enzyme sequences for building the ECemble method. Pfam (Protein Family) database contains functional domain information for protein sequences; Prosite database is a collection of biologically important sites, patterns and sequence motifs associated with protein functions; and the Superfamily database contains sets of homologous proteins that conserve structural and active site features. Together, these three databases provide a comprehensive coverage of functional, active site and structure-based features of enzyme and non-enzyme protein sequences.

\section{Machine learning methods}

We selected five diverse and most popular machine learning classifiers; Naïve Bayes [34], k-Nearest Neighbor (KNN) [35] classifier, Support Vector Machine (SVM) [36], Decision Stump (DS) [41] and Random Forest (treebased) classifiers (RFC) [37], to build models for enzyme identification and classification. We used the WEKA 3.7.5 [42] package, which is an open-source, Java-based framework to build classification models using different ML techniques.

\section{Ten proteome datasets}

We downloaded the proteomes of ten model organisms from the SwissProt database [45], ranging from animal, plant, fungal and bacterial species such as Saccharomyces cerevisiae (yeast), Caenorhibditis elegans (C. elegans), Drosophila melanogaster (fruitfly), Danio rerio (zebrafish), Gallus gallus (chicken), Mus musculus (mouse), Homo sapiens (human), Escherichia coli (E. coli), Oryza sativa (rice) and Arabidopsis thaliana (Arabidopsis). We filtered out the experimentally known enzyme sequences from each of the ten proteomes and tested the unclassified sequences with ECemble.

\section{Human gut metagenomic samples}

We used a study on the human gut microbiome from the Beijing Genomics Institute's (BGI) Metagenomic

Table 7. Class-wise statistics on the number of enzyme sequences, and the subclasses at each level

\begin{tabular}{|c|c|c|c|c|}
\hline $\begin{array}{l}\text { EC Level-1(6 Classes) } \\
\text { (Example:1.x.x.x) }\end{array}$ & Total Sequences & $\begin{array}{c}\text { Subclasses at } \\
\text { EC Level-2 (example:1.1.x.x) }\end{array}$ & $\begin{array}{c}\text { Subclasses at } \\
\text { EC Level-3 (example:1.1.1.x) }\end{array}$ & $\begin{array}{c}\text { Subclasses at } \\
\text { EC Level-4 (example:1.1.1.1) }\end{array}$ \\
\hline EC1: Oxidoreductases & 8662 & 22 & 90 & 658 \\
\hline EC2: Transferases & 23604 & 9 & 31 & 751 \\
\hline EC3: Hydrolases & 15183 & 11 & 49 & 781 \\
\hline EC4: Lyases & 5525 & 7 & 15 & 268 \\
\hline EC5: Isomerases & 4146 & 6 & 17 & 127 \\
\hline EC6: Ligases & 7830 & 6 & 11 & 109 \\
\hline Total & 64950 & 61 & 213 & 2693 \\
\hline
\end{tabular}

There are 6 major classes at Level-1 that expand into 61, 213 and 2,693 subclasses at Levels 2, 3 and 4, respectively. 
Sequencing Project [26] that describes a set of about 3.3 million microbial genes sequenced and assembled from fecal samples of 124 individuals (Additional file 18 represent the patient profiles and health status), to study the impact of human gut microbiome on human metabolism. As part of the filtering steps, sequences mapped to viruses, archaea, eukaryota, unclassified and unknown sequences were removed. About 2.5 million translated protein sequences from the assembled scaffolds of bacterial genomes were predicted by our ECemble method to identify all the bacterial enzymes in the human gut microbiome. The diversity of taxonomic groups of bacteria at each level is given in Additional file 19: Figure S5.

\section{KEGG database}

We used Kyoto Encyclopedia of Genes and Genomes (KEGG) [46], the most comprehensive database source that integrates genomic, proteomic and systemic functional information for pathway analysis. The KEGG Pathway suite is a collection of manually drawn maps demonstrating the existing knowledge on the molecular interaction and reaction networks. KEGG pathways were used as reference pathways to map human and gut metagenomic enzymes for analysis of gut microbecomplemented human metabolic pathways.

\section{Performance measurements}

We used standard evaluation metrics that include 10-fold cross validation and ROC curves. In 10-fold cross-validation, sequences at each level are divided into ten parts, models are built using nine parts, and predictions are generated and evaluated on the data contained in one part. This procedure is repeated ten times, where each part is tested against the models built from nine other parts. The average performance of the ten models is considered as an unbiased estimate of the training model performance. After cross-validation, we assessed the performance of the fully trained classifier models using the test set $(20 \%$ of original data) that were hidden from the classifiers. We report standard performance measures over each enzyme class including the following: true positives (TP) as the number of sequences that are correctly identified in a class that belongs to them; false negatives (FN) as the number of sequences that are not identified in a class that belongs to them; true negatives (TN) as the number of sequences that are not found in a class that does not belong to them; false positives (FP) as the number of sequences that are identified in a class that does not belong to them; sensitivity as the proportion of true positives that are predicted as positives; specificity is the proportion of true negatives that are predicted as negatives. The sensitivity and specificity are given by, sensitivity $=\mathrm{TP} /(\mathrm{TP}+\mathrm{FN})$; specificity $=$ $\mathrm{TN} /(\mathrm{TN}+\mathrm{FP})$.
We also report accuracy in a class as the ratio of the number of correctly predicted enzyme sequences to the total number of sequences in that class. We optimize and validate the accuracy of ECemble by selecting the optimal model(s) that has maximum true positive rate (sensitivity) and minimum false positive rate (1-specificity). Finally, we show ROC (Receiver Operating Characteristic) curves as a graphical means of measuring the performance for each class at each level of the prediction process, and the area under the curve (AUC) as a numeric measure of performance depicted by ROC curves.

\section{Additional material}

Additional file 1: Figure S1. 10-fold cross validation and testing accuracies. A) For enzyme identification at EC Level-0 using ML classifiers Decision Stump (DS), Naïve Bayes Classifier (NBC), K-Nearest Neighbor (KNN), Support Vector Machine (SVM), and Random Forest Classifier (RFC). B) For enzyme classification at EC Level-1 using ML classifiers Decision Stump (DS), Naïve Bayes Classifier (NBC), K-Nearest Neighbor (KNN), Support vector Machine (SVM), and Random Forest Classifier (RFC). Additional file 2: Table S1. Overall predictions of 2 classifiers vs. 3 classifiers.

Additional file 3: Figure S2. Accuracy and distribution of enzyme sequence and class. A) Distribution of enzyme sequence and class coverage for cdh70, cdh60, cdh50 and cdh40 datasets. B) Accuracy at each EC level for cdh70, cdh60, cdh50 and cdh40 datasets.

Additional file 4: Table S2. Accuracy of ECemble method using cdh70, cdh60, cdh50 and cdh40 datasets

Additional file 5: Statistics for Known and ECemble predicted enzyme sequences from ten proteomes. The proteomes contains both reviewed and unreviewed sequences from UniProt (Data Sheet 'ReviewedAndUnreviewed'). The ECemble predictions for the reviewed sequences from 10 proteomes are given in Data Sheet

'PredictionsReviewedSet'.

Additional file 6: ECemble predicted gut microbial enzymes. Additional file 7: Known human enzyme reactions from SwissProt and KEGG.

Additional file 8: Figure S3. GMC pathways that validate the role of gut bacteria in human metabolism

Additional file 9: Figure S4. GMC pathways that reveal the newly discovered role of gut bacteria in human metabolism

Additional file 10: Mapped human pathways in major pathway categories.

Additional file 11: CAZymes found in metagenomic samples using ECemble.

Additional file 12: Known enzymes common in both human and bacteria.

Additional file 13: Known bacterial enzyme reactions in the gut microflora predicted by ECemble

Additional file 14: Unknown gut bacterial enzyme reactions that are predicted by ECemble.

Additional file 15: ECemble predicted gut bacterial enzymes with subject information.

Additional file 16: Obesity/Lean enzymes from metagenomic samples using Fisher-exact test. Obese_Normalized is calculated as the ratio of number of obese subjects having a particular enzyme to the total number of obese subjects (42). Similarly, Lean_Normalized is calculated as the ratio of number of lean subjects having a particular enzyme to the total number of lean subjects (82). 
Additional file 17: IBD/Non-IBD enzymes from metagenomic samples using Fisher-exact test. IBD_Normalized is calculated as the ratio of number of IBD subjects having a particular enzyme to the total number of IBD subjects (25). Similarly, Non-IBD_Normalized is calculated as the ratio of number of Non-IBD subjects having a particular enzyme to the total number of Non-IBD subjects (99).

\section{Additional file 18: Metagenomic samples profile.}

Additional file 19: Figure S5. Taxonomic distribution of bacteria species from metagenomic samples.

\section{List of abbreviations used}

AUC: Area Under Curve

DS: Decision Stump

EC: Enzyme Commission

IBD: Inflammatory Bowel Disease

KNN: K-Nearest Neighbor

KEGG: Kyoto Encyclopedia of Genes and Genomes

ML: Machine Learning

NBC: Naïve Bayes Classifier

RFC: Random Forest Classifier

ROC: Receiver Operating Characteristic

SF: Superfamily

SVM: Support Vector Machine

WEKA: Waikato Environment for Knowledge Analysis

\section{Competing interests}

The authors declare that they have no competing interests.

\section{Authors' contributions}

AM developed the ECemble method using machine-learning approaches and applied it to analyze gut metabolic pathways, and drafted the manuscript. CG conceived the idea of the project, provided overall guidance and coordination, suggested improvement and corrected the manuscript. All authors read and approved the final version of the manuscript.

\section{Acknowledgements}

This research has been partly supported by National Institutes of Health [1R01GM086533-01A1 to CG], startup funds to CG from UNMC, and the graduate fellowship from UNMC to AM. The publication costs for this article have been funded by the startup funds to CG from UNMC.

This article has been published as part of BMC Genomics Volume 16 Supplement 7, 2015: Selected articles from The International Conference on Intelligent Biology and Medicine (ICIBM) 2014: Genomics. The full contents of the supplement are available online at http://www.biomedcentral.com/ bmcgenomics/supplements/16/S7.

\section{Authors' details}

'Department of Genetics, Cell Biology and Anatomy, University of Nebraska Medical Center, Omaha, NE, 68198, USA. ${ }^{2}$ Bioinformatics and Systems Biology Core, University of Nebraska Medical Center, Omaha, NE, 68198, USA. ${ }^{3}$ Department of Biochemistry and Molecular Biology, University of Nebraska Medical Center, Omaha, NE, 68198, USA. ${ }^{4}$ Fred and Pamela Buffet Cancer Center, University of Nebraska Medical Center, Omaha, NE, 68198, USA.

Published: 11 June 2015

\section{References}

1. Jeremy MB, John LT, Lubert S: Biochemistry W.H. Freeman; 1988, 444-445

2. Schmidt S: Metabolites: a helping hand for pathway evolution? Trends Biochem Sci 2003, 28:336-341.

3. Shah I, Hunter L: Predicting enzyme function from sequence: a systematic appraisal. Proc International Conf Intell Syst Mol BiolISMBInternational Conf Intell Syst Mol Biol 1997, 5:276-283.

4. Webb EC: Enzyme Nomenclature San Diego: Academic Press; 1992

5. Tian W, Skolnick J: How well is enzyme function conserved as a function of pairwise sequence identity? J Mol Biol 2003, 333:863-882.
6. Espadaler J, Eswar N, Querol E, Avilés FX, Sali A, Marti-Renom M a, Oliva B: Prediction of enzyme function by combining sequence similarity and protein interactions. BMC Bioinformatics 2008, 16:249.

7. Cheng BYM, Carbonell JG, Klein-Seetharaman J: Protein classification based on text document classification techniques. Proteins 2005, 58:955-970.

8. McCallum a, Nigam K: A comparison of event models for naive bayes text classification. Work Learn Text Categ 1998, 41-48.

9. Mitchell TM: Machine Learning Boston 1997, 4:432.

10. Duda R, Hart P, Stork D: Pattern Classification 2001, 680

11. Mohammed A, Guda C: Computational Approaches for Automated Classification of Enzyme Sequences. J Proteomics Bioinforma 2011, 04:147-152.

12. Chou KC: Using amphiphilic pseudo amino acid composition to predict enzyme subfamily classes. Bioinformatics 2005, 21:10-19.

13. Otto TD, Guimarães ACR, Degrave WM, de Miranda AB: AnEnPi: identification and annotation of analogous enzymes. BMC Bioinformatics 2008, 16:544.

14. Röttig $M$, Rausch $C$, Kohlbacher $O$ : Combining structure and sequence information allows automated prediction of substrate specificities within enzyme families. PLoS Comput Biol 2010, 6:e1000636.

15. Almonacid DE, Babbitt PC: Toward mechanistic classification of enzyme functions. Curr Opin Chem Biol 2011, 15:435-442.

16. Lu L, Qian Z, Cai YD, Li Y: ECS: an automatic enzyme classifier based on functional domain composition. Comput Biol Chem 2007, 31:226-32.

17. Shen HB, Chou KC: EzyPred: a top-down approach for predicting enzyme functional classes and subclasses. Biochem Biophys Res Commun 2007, 364:53-9.

18. Weingart $U$, Lavi $Y$, Horn D: Data mining of enzymes using specific peptides. BMC Bioinformatics 2009, 10:446.

19. Nasibov E, Kandemir-Cavas C: Efficiency analysis of KNN and minimum distance-based classifiers in enzyme family prediction. Comput Biol Chem 2009, 33:461-4.

20. Shi $R$, Hu X: Predicting enzyme subclasses by using support vector machine with composite vectors. Protein Pept Lett 2010, 17:599-604.

21. Wang YC, Wang Y, Yang ZX, Deng NY: Support vector machine prediction of enzyme function with conjoint triad feature and hierarchical context. BMC Syst Biol 2011, 5(Suppl 1):S6

22. Borro LC, Oliveira SRM, Yamagishi MEB, Mancini AL, Jardine JG, Mazoni I, Santos EH Dos, Higa RH, Kuser PR, Neshich G: Predicting enzyme class from protein structure using Bayesian classification. Genet Mol Res 2006, 5:193-202

23. Hung SS, Wasmuth J, Sanford C, Parkinson J: DETECT-a density estimation tool for enzyme classification and its application to Plasmodium falciparum. Bioinformatics 2010, 26:1690-8.

24. Arakaki AK, Huang Y, Skolnick J: EFICAz2: enzyme function inference by a combined approach enhanced by machine learning. BMC Bioinformatics 2009, 10:107.

25. Syed U, Yona G: Enzyme function prediction with interpretable models. Methods Mol Biol 2009, 541:373-420.

26. Qin J, Li R, Raes J, Arumugam M, Burgdorf KS, Manichanh C, Nielsen T, Pons N, Levenez F, Yamada T, Mende DR, Li J, Xu J, Li S, Li D, Cao J, Wang $B$, Liang $H$, Zheng $H$, Xie Y, Tap J, Lepage $P$, Bertalan M, Batto JM Hansen T, Le Paslier D, Linneberg A, Nielsen HB, Pelletier E, Renault P, et al: A human gut microbial gene catalogue established by metagenomic sequencing. Nature 2010, 464:59-65.

27. Bäckhed F: Host responses to the human microbiome. Nutr Rev 2012 70(Suppl 1):S14-7.

28. Cummings $\mathrm{JH}$ : Microbial digestion of complex carbohydrates in man Proc Nutr Soc 1984, 43.35-44.

29. Hooper L V, Midtvedt T, Gordon Jl: How host-microbial interactions shape the nutrient environment of the mammalian intestine. Annu Rev Nutr 2002, 22:283-307.

30. Kelly D, Campbell Jl, King TP, Grant G, Jansson EA, Coutts AGP, Pettersson S, Conway S: Commensal anaerobic gut bacteria attenuate inflammation by regulating nuclear-cytoplasmic shuttling of PPAR-gamma and RelA. Nat Immunol 2004, 5:104-112.

31. Greenblum S, Turnbaugh PJ, Borenstein E: Metagenomic systems biology of the human gut microbiome reveals topological shifts associated with obesity and inflammatory bowel disease. Proc Natl Acad Sci USA 2012, 109:594-9. 
32. Nicholson JK, Holmes E, Kinross J, Burcelin R, Gibson G, Jia W, Pettersson S: Host-gut microbiota metabolic interactions. Science 2012, 336:1262-7.

33. Lepage $P$, Leclerc $M C$, Joossens $M$, Mondot $S$, Blottière HM, Raes J, Ehrlich D, Doré J: A metagenomic insight into our gut's microbiome. Gut 2013, 62:146-58.

34. Rish I: An empirical study of the naive Bayes classifier. IJCAI 2001 Work Empir Methods Artif Intell 2001, 3:41-46.

35. Cover T, Hart P: Nearest neighbor pattern classification. IEEE Trans Inf Theory 1967, 13:21-27.

36. Cortes C, Vapnik V: Support-vector networks. Mach Learn 1995, 20:273-297.

37. Breiman L: Random Forests. Mach Learn 2001, 45:5-32.

38. Bateman A, Birney E, Durbin R, Eddy SR, Finn RD, Sonnhammer EL: Pfam 3.1: 1313 multiple alignments and profile HMMs match the majority of proteins. Nucleic Acids Res 1999, 27:260-262.

39. Wilson D, Madera M, Vogel C, Chothia C, Gough J: The SUPERFAMILY database in 2007: families and functions. Nucleic Acids Res 2007, 35: D308-D313.

40. Bairoch A, Bucher P, Hofmann K: The PROSITE database, its status in 1997. Nucleic Acids Res 1997, 25:217-221.

41. Iba W, Langley P: Induction of One-Level Decision Trees. ML92 Proc Ninth Int Conf Mach Learn Aberdeen, Scotland, 1-3 July 1992 San Francisco, CA: Morgan Kaufmann; 1992, 233-240.

42. Hall M, Frank E, Holmes G, Pfahringer B, Reutemann P, Witten $\mathbb{H}$ : The WEKA data mining software: an update. SIGKDD Explor 2009, 11:10-18.

43. Li W, Godzik A: Cd-hit: a fast program for clustering and comparing large sets of protein or nucleotide sequences. Bioinformatics 2006, 22:1658-1659.

44. Magrane $\mathrm{M}$, Consortium U: UniProt Knowledgebase: a hub of integrated protein data. Database (Oxford) 2011, 2011:bar009.

45. Bairoch A, Apweiler R: The SWISS-PROT protein sequence database and its supplement TrEMBL in 2000. Nucleic Acids Res 2000, 28:45-48.

46. Kanehisa M, Goto S: KEGG: kyoto encyclopedia of genes and genomes. Nucleic Acids Res 2000, 28:27-30.

47. Abratt VR, Reid SJ: Oxalate-degrading bacteria of the human gut as probiotics in the management of kidney stone disease. Adv Appl Microbiol 2010, 72:63-87.

48. Blaut M, Clavel T: Metabolic diversity of the intestinal microbiota: implications for health and disease. J Nutr 2007, 137:751S-5S.

49. Dumas ME, Barton RH, Toye A, Cloarec O, Blancher C, Rothwell A, Fearnside J, Tatoud R, Blanc V, Lindon JC, Mitchell SC, Holmes E, McCarthy MI, Scott J, Gauguier D, Nicholson JK: Metabolic profiling reveals a contribution of gut microbiota to fatty liver phenotype in insulinresistant mice. Proc Natl Acad Sci USA 2006, 103:12511-6.

50. Clayton TA, Baker D, Lindon JC, Everett JR, Nicholson JK: Pharmacometabonomic identification of a significant host-microbiome metabolic interaction affecting human drug metabolism. Proc Natl Acad Sci USA 2009, 106:14728-14733.

51. Sousa T, Paterson R, Moore V, Carlsson A, Abrahamsson B, Basit AW: The gastrointestinal microbiota as a site for the biotransformation of drugs. Int J Pharm 2008, 1-25.

52. Cani PD, Amar J, Iglesias MA, Poggi M, Knauf C, Bastelica D, Neyrinck AM, Fava F, Tuohy KM, Chabo C, Waget A, Delmée E, Cousin B, Sulpice T, Chamontin B, Ferrières J, Tanti JF, Gibson GR, Casteilla L, Delzenne NM, Alessi MC, Burcelin R: Metabolic endotoxemia initiates obesity and insulin resistance. Diabetes 2007, 56:1761-1772.

53. Turnbaugh PJ, Henrissat B, Gordon Jl: Viewing the human microbiome through three-dimensional glasses: integrating structural and functional studies to better define the properties of myriad carbohydrate-active enzymes. Acta Crystallogr Sect F Struct Biol Cryst Commun 2010, 66(Pt 10):1261-1264.

54. Devaraj S, Hemarajata P, Versalovic J: The human gut microbiome and body metabolism: implications for obesity and diabetes. Clin Chem 2013, 59:617-28

55. Cantarel BL, Lombard V, Henrissat B: Complex carbohydrate utilization by the healthy human microbiome. PLoS One 2012, 7:e28742.

56. R Development Core Team: R: A Language and Environment for Statistical Computing. R Found Stat Comput Vienna Austria 2013, \{ISBN\} 3900051-07-0.

57. Sanderson IR, Boulton P, Menzies I, W-SJ : Improvement of abnormal lactulose / rhamnose permeability in active Crohn 's disease of the small bowel by an elemental diet. Gut 1987, 28(February):1073-1076.
58. Desai D, Faubion WA, Sandborn WJ: Review article: biological activity markers in inflammatory bowel disease. Aliment Pharmacol Ther 2007, 25:247-255

59. Bairoch A: The ENZYME database in 2000. Nucleic Acids Res 2000, 28:304-305.

60. Johnson LS, Eddy SR, Portugaly E: Hidden Markov model speed heuristic and iterative HMM search procedure. BMC Bioinformatics 2010, 11:431.

61. de Castro E, Sigrist CJA, Gattiker A, Bulliard V, Langendijk-Genevaux PS, Gasteiger E, Bairoch A, Hulo N: ScanProsite: detection of PROSITE signature matches and ProRule-associated functional and structural residues in proteins. Nucleic Acids Res 2006, 34:W362-W365.

doi:10.1186/1471-2164-16-S7-S16

Cite this article as: Mohammed and Guda: Application of a hierarchica enzyme classification method reveals the role of gut microbiome in human metabolism. BMC Genomics 2015 16(Suppl 7):S16.

\section{Submit your next manuscript to BioMed Central and take full advantage of:}

- Convenient online submission

- Thorough peer review

- No space constraints or color figure charges

- Immediate publication on acceptance

- Inclusion in PubMed, CAS, Scopus and Google Scholar

- Research which is freely available for redistribution

Submit your manuscript at www.biomedcentral.com/submit
C Biomed Central 